\title{
Facile synthesis of fluorescent carbon nanodots from starch nanoparticles
}

\author{
Suk Fun Chin ${ }^{a, *}$, Siti Nur Akmar Mohd Yazid ${ }^{a}$, Suh Cem Pang ${ }^{a}$, Sing Muk Ng ${ }^{b}$ \\ ${ }^{a}$ Faculty of Resource Science and Technology, Universiti Malaysia Sarawak, 94300 Kota Samarahan, Sarawak, Malaysia \\ ${ }^{\mathrm{b}}$ School of Engineering, Computing, and Science, Swinburne University of Technology Sarawak Campus, 93350 Kuching, Sarawak, Malaysia
}

\section{A R T I C L E I N F O}

\section{Article history:}

Received 21 March 2012

Accepted 23 June 2012

Available online 2 July 2012

\section{Keywords:}

Carbon nanodots

Fluorescent

Starch nanoparticles

\begin{abstract}
A B S T R A C T
Fluorescent carbon dots were synthesized by the carbonization of preformed native sago starch nanoparticles, followed by surface oxidation in an aqueous medium. The morphology and particle sizes of carbon nanodots (CDs) were observed to be very similar to starch nanoparticles that were used as precursors. The particle sizes of CDs synthesized in this study were within the range 50-80 nm, which were much larger than those of very tiny CDs $(1-5 \mathrm{~nm})$ previously reported. The CDs portrayed fluorescent with constant emission wavelength peak at $430 \mathrm{~nm}$ when excited with various higher energy photons. The non-shifting of the emission wavelength peak suggested that CDs prepared in this study were highly homogenous and possessed mono-dispersed physiochemical properties.
\end{abstract}

(c) 2012 Elsevier B.V. All rights reserved

\section{Introduction}

Fluorescent semiconductor quantum dots (QDs), such as CdSe and CdTe have received numerous attentions due to their promising potential applications in biomedical field especially in biosensing and bioimaging applications. However, the intrinsic toxicity, high cost and complicated preparation methods of QDs have limited their practical applications in biomedical fields. Recently, carbon nanodots (CDs) have emerged as a new class of carbon based fluorescent nanoparticles. Due to their potentially low toxicity, potential biocompatibility, low cost and interesting fluorescent properties, CDs hold great promise to be an alternative to QDs. Some synthesis methods of CDs have been proposed in literatures. CDs have been prepared from carbon nanotubes (CNTs) through an electrochemical method [1]. Hu et al. prepared $4-5 \mathrm{~nm}$ of CDs through laser irradiation of carbon powders in organic solvents [2]. Very tiny CDs $(1 \mathrm{~nm})$ were prepared by combustion of candle soot, followed by refluxed with $\mathrm{HNO}_{3}$ for $12 \mathrm{~h}$ [3]. More recently, some researchers have prepared CDs by using carbohydrates as starting materials, followed by surface oxidation by refluxed with $\mathrm{HNO}_{3}$ for $12 \mathrm{~h}$. Fluorescent CDs were also prepared by using watermelon peels as carbon sources [4]. However, most of these synthetic methods involved expensive starting materials (e.g. CNTs), complicated instruments, prolong reflux, and can have less control over the properties of the produced CDs.

In this paper, we report a simple, fast and aqueous based synthesis approach to prepare CDs by using preformed starch

\footnotetext{
${ }^{*}$ Corresponding author. Tel.: +60 82582999.

E-mail addresses: sfchin@frst.unimas.my, sukfunchin@gmail.com (S.F. Chin).
}

nanoparticles as precursor materials. Sago starch that is locally available and cheap was used as raw materials to prepare starch nanoparticles. Starch nanoparticles of around $50-80 \mathrm{~nm}$ were converted to $\mathrm{CDs}$ by dehydration using concentrated $\mathrm{H}_{2} \mathrm{SO}_{4}$, followed by oxidation with $\mathrm{HNO}_{3}$ for $30 \mathrm{~min}$. To the best of our knowledge, this is the first report on using starch nanoparticles as precursor materials for CDs synthesis. Due to the small size and high surface areas of starch nanoparticles that were used as precursor materials, we were able to reduce the surface oxidation process duration to only $30 \mathrm{~min}$, while produced CDs that are highly homogenous.

\section{Experimental}

\subsection{Materials}

All chemicals were of reagent grade and were used without further purification. Ultra pure water $\left(\sim 18.2 \mathrm{M} \Omega, 25^{\circ} \mathrm{C}\right)$ was obtained from a Milipore Mili-Q system. Native sago starch powder was obtained from a local grocery store.

\subsection{Sample preparation}

Starch nanoparticles were prepared from locally available native sago starch based on our previously reported synthesis method [4]. Starch nanoparticles were converted to carbon nanoparticles by dehydration with concentrated $\mathrm{H}_{2} \mathrm{SO}_{4}$ [5]. In a typical synthesis, $2.0 \mathrm{~g}$ of starch nanoparticles were dispersed in $5 \mathrm{~mL}$ of ultrapure water and $8.0 \mathrm{ml}$ of concentrated $\mathrm{H}_{2} \mathrm{SO}_{4}$ was added to the starch solution. The reaction was allowed to proceed for $40 \mathrm{~min}$, followed by the addition of $40 \mathrm{~mL}$ of ultra pure water. The black carbon 